\section{Cooperativity in the dislocation theory of melting}

THE interesting behaviour of the shear moduli of alkali halides in the vicinity of the melting transition was recently noted by Tallon, Robinson and Smedley ${ }^{1}$. These parameters do not fall to zero at the crystal melting temperature, $T_{m}$, as proposed by Born ${ }^{2}$, but a zero value is attained on extrapolating to the dilation corresponding to the liquid at $T_{\mathrm{m}}$. It is suggested that 'melting occurs when a solid can transform isothermally to a state of zero shear modulus', and that a two-phase melting model follows. Although they do not deal with the microscopic processes occurring along the melting isotherm their discussion does have a bearing on the important role of cooperative effects. We comment here both on the specific points raised by their analysis and the question of cooperativity in the dislocation theory of melting.

The extrapolation by Tallon et al. would not be possible for crystals showing a negative volume change of melting. Such materials, nevertheless, exhibit continuous expansion right up to $T_{\mathrm{m}}$. Also a plot of shear modulus against volume causes temperature to become a 'hidden variable' for the region below $T_{\mathrm{m}}$. The success of the Tallon et al. extrapolation suggests, however, that, for the alkali halides at least, the volume change is the dominating influence on the shear modulus.

Mizushima $^{3}$ (and recently Edwards ${ }^{4}$ ) showed that because dislocation energy, $E_{\mathrm{d}}$, depends on dislocation concentration, $C_{\mathrm{d}}$, the free energy of a crystal will vary with $C_{\mathrm{d}}$ (used as an order parameter) in the manner described by Landau and Lifschitz ${ }^{5}$ for a first order transition; for $T<T_{m}$ equilibrium prevails at $C_{\mathrm{d}}=0$, but $C_{\mathrm{d}}$ suddenly goes to its saturation value at $T=T_{\mathrm{m}}$. Further insight into the physical basis of this behaviour is obtained by noting ${ }^{6}$ that at equilibrium $C_{\mathrm{d}}=\exp$ [ $\left.-\left(E_{\mathrm{d}}-T S_{\mathrm{d}}\right) / k T\right], S_{\mathrm{d}}$ being dislocation entropy, but that $E_{\mathrm{d}}=$ $-\left(G b^{2} / 8 \pi\right) \ln C_{\mathrm{d}} \delta^{2}$, where $\delta<1$ and $C_{\mathrm{d}}$ is given as an atomic fraction ${ }^{7},(G$ and $b$ are the shear modulus and Burgers vector, respectively). Thus, $C_{\mathrm{d}}$ depends on $T$ in a 'super-Arrhenius' manner, having a non-zero equilibrium value only at a temperature $T^{*}=-b^{3} G\left(\ln \delta^{2}\right) / 40 \pi k$, the limit of stability in the infinite crystal, which Mizushima identifies as $T_{\mathrm{m}}$. Kuhlmann-Wilsdorf has extended this approach and shown that it leads to impressive agreement between experimental melting points and values calculated from elastic constants and crystallographic data $^{8}$. Taking a lead from Tallon et al. ${ }^{1}$, we point out that because a dislocated lattice dilates ${ }^{9}, G$ will fall as $C_{\mathrm{d}}$ increases. This additional factor will make $C_{\mathrm{d}}$ depend even more critically on $T$ in the vicinity of $T^{*}$. Moreover, dilation propagates through the crystal at the sound velocity so this cooperativity is potentially stronger than the essentially positional factor arising in Mizushima's model. Finally, even $S_{\mathrm{d}}$ will vary with $C_{\mathrm{d}}$ through dilation-induced changes in the eigen frequencies.

Dislocations generated inside a crystal by thermal fluctuations, either at or below $T_{\mathrm{m}}$, must, from topological requirements, be dipoles $(+b$ and $-b)$ as predicted by KuhlmannWilsdorf ${ }^{8}$, and subsequently observed ${ }^{10,11}$. These are favoured by the $C_{\mathrm{d}}$ dependence of $E_{\mathrm{d}}$ because the two dislocations cancel each other's strain field and only the core energy is left (that is, $E_{\mathrm{d}}$ simply becomes the core energy). This only applies as long as they are a bound pair, so the inevitable fate of a dipole below $T_{\mathrm{m}}$ is mutual annihilation. Furthermore, the influence of such transient dipoles on a crystal's viscosity is not just small but identically zero because the effect of one dislocation on the lattice will always be exactly counteracted by the second member of the dipole pair. The saturation (and instability) situation is reached when the inter-dipole distance equals the spacing of the dislocations in the dipoles (about a core diameter), which is analogous to the Debye screening length ${ }^{4}$.

In practice, crystals melt at their surfaces, and the influence of a bounding interface (solid-gas or solid-liquid) has been discussed in both continuum ${ }^{12}$ and atomistic ${ }^{13.14}$ models. In particular, Tallon ${ }^{13}$ shows how a surface can interfere with mutual annihilation in dipole pairs by absorbing a dislocation. Energetic considerations also show that the surface functions as a dislocation source and becomes a natural nucleation site for the cooperative process discussed above. The surface has, in effect, a melting point, $T_{\mathrm{sm}}$, which lies lower than Mizushima's $T^{*}$, and corresponds to the situation in which the dislocation saturation condition is attained in a layer of minimal thickness (presumably equal to the dislocation core diameter). The picture of melting that emerges from these considerations is as follows: between $T_{\mathrm{sm}}$ and $T^{*}$ dynamic equilibrium between dislocation generation and annihilation leaves the interior dislocation free but permits a gradual rise to the saturation dislocation density at the surface. Immediately above $T^{*}$ the crystal-liquid interface advances into the interior at a speed that is predominantly determined by the dipole generation rate.

\section{R. M. J. COTTERILL}

Department of Structural Properties of Materials,

The Technical University of Denmark,

DK-2800 Lyngby, Denmark

Received 18 November 1977; accepted 5 April 1978.

1. Tallon, J. L., Robinson, W. H. \& Smedley, S. I. Nature 266, 337 (1977). 2. Born, M. J. chem. Phys. 7, 591 (1939).

Mizushima, S. J. phys. Soc. Japan 15, 70 (1960)

Landau, L. D. \& Lifschitz, E. M. Statistical Physics, 426 (Pergamon, Oxford, 1970)

Friedel, J. Dislocations, 75 (Pergamon, Oxford, 1964).

7. Nabarro, F. R. N. Theory of Crystal Dislocations, 688 (Oxford University Press, 1967)

Kuhlmann-Wilsdorf, D. Phys. Rev. 145, 465 (1966)

9. Nabarro, F. R. N. Theory of Crystal Dislocations, 166 (Oxford University Press, 1967).

0. Cotterill, R. M. J. \& Pedersen, L. B. Solid St. Commun. 10, 439 (1972) 11. Cotterill, R. M. J., Damgaard Kristensen, W. \& Jensen, E. J. Phil. Mag. 30, 245 (1974)

Klæstrup Kristensen, J. \& Cotterill, R. M. J. Phil. Mag. 36, 437 (1977) 13. Tallon, J. L. thesis, Victoria Univ. Wellington, New Zealand (1976) 4. Cotterill, R. M. J. Phil. Mag. 32, 1283 (1975).

\section{Uncatalysed oscillatory chemical reactions}

THE most widely studied oscillatory chemical reaction is the catalytic oxidation of a variety of organic compounds by acidic bromate--the Belousov-Zhabotinsky reaction. Babu et al. ${ }^{1}$ have reported that the gallic acid, bromate, sulphuric acid and cobalt (II) system showed oscillatory behaviour. They claim that the two key steps are the oxidation of cobalt (II) by bromate to cobalt (III), and the reduction of the latter by gallic acid (GA), that is, they regarded the chemical process as a BelousovZhabotinsky-type oscillatory reaction. Redox potential data on the $\mathrm{Co}^{3+} / \mathrm{Co}^{2+}(1.81 \mathrm{~V}), \mathrm{BrO}_{3}{ }^{-} / \mathrm{Br}^{-}(1.44 \mathrm{~V})$ and $\mathrm{BrO}_{3}{ }^{-} / \mathrm{Br}_{2}$ $(1.51 \mathrm{~V})$ systems, imply that the reduction of bromate by cobalt (II) is highly unfavourable thermodynamically which made us sceptical about the suggested steps. We report here our preliminary investigations mostly on the $\mathrm{GA}-\mathrm{BrO}_{3}--\mathrm{H}_{2} \mathrm{SO}_{4}$ system which attempted to unravel this problem. One of our first experiments showed that chemical oscillation occurred even in the absence of a catalyst. This unexpected finding indicated novel perspectives regarding chemical oscillators.

Adding bromate to a stirred mixture of $\mathrm{GA}$ and $\mathrm{H}_{2} \mathrm{SO}_{4}$ immediately turns the solution dark brown, and after a certain time suddenly to yellow. This is followed by repeated colour changes from brown to yellow with a decreasing frequency. The oscillatory behaviour of the system was traced with a $\mathrm{Pt}$ electrode or with a bromide selective sensor; $\mathrm{Hg} / \mathrm{Hg}_{2} \mathrm{SO}_{4} / \mathrm{K}_{2} \mathrm{SO}_{4}$ was used as a reference electrode. Periodicity was found also in the rate of heat evolution similar to that observed with the Belousov-Zhabotinsky systems ${ }^{2-4}$. Chemical oscillations were looked at in the following concentration ranges: 0.025 $0.05 \mathrm{M} \mathrm{GA}, 0.04-0.075 \mathrm{M} \mathrm{KBrO}_{3}$, and above $1.5 \mathrm{M} \mathrm{H}_{2} \mathrm{SO}_{4}$ and $1.5 \mathrm{M} \mathrm{HNO}_{3}$, respectively. (All the chemicals used were of analytical grade with heavy metal content below 5 p.p.m. checked by atomic absorption spectrophotometry.) Typical curves of redox potential and bromide concentration oscillations are shown in Fig. 1. The temperature change during the reaction is seen in Fig. 2. A few data on the length of time of the first 